\title{
Extremely high quantum efficiency of donor-acceptor-pair emission in $\mathrm{N}$-and-B-doped $6 \mathrm{H}-\mathrm{SiC}$
}

\section{$\operatorname{AUTHOR}(S)$ :}

Kamiyama, S; Maeda, T; Nakamura, Y; Iwaya, M; Amano, H; Akasaki, I; Kinoshita, H; ... Monemar, B; Onuma, T; Chichibu, SF

\section{CITATION:}

Kamiyama, S ...[et al]. Extremely high quantum efficiency of donor-acceptor-pair emission in N-and-B-doped 6H-SiC. Journal of Applied Physics 2006, 99(9): 093108.

\section{ISSUE DATE:}

2006-05-01

URL:

http://hdl.handle.net/2433/24210

\section{RIGHT:}

Copyright 2006 American Institute of Physics. This article may be downloaded for personal use only. Any other use requires prior permission of the author and the American Institute of Physics. 


\title{
Extremely high quantum efficiency of donor-acceptor-pair emission in $\mathrm{N}$-and-B-doped $6 \mathrm{H}$-SiC
}

\author{
S. Kamiyama, ${ }^{\text {a) }}$ T. Maeda, Y. Nakamura, M. Iwaya, H. Amano, and I. Akasaki \\ Faculty of Science and Technology and Nano Factory, Meijo University, 1-501 Shiogamaguchi, \\ Tempaku-ku, Nagoya 468-8502, Japan \\ $\mathrm{H}$. Kinoshita and T. Furusho \\ SiXON Ltd., Takasecho, Ukyo-ku, Kyoto, Japan \\ M. Yoshimoto \\ Cooperative Research Center, Kyoto Institute of Technology, Matsugasaki, Sakyo-ku, \\ Kyoto 606-8585, Japan \\ T. Kimoto and J. Suda \\ Department of Electronic Science and Engineering, Kyoto University, Kyoto-daigaku Katsura, \\ Nishigyo-ku, Kyoto 615-8510, Japan
}

A. Henry, I. G. Ivanov, J. P. Bergman, and B. Monemar

Department of Physics and Measurement Technology, Linköping University, 58183 Linköping, Sweden

T. Onuma and S. F. Chichibu

Institute of Applied Physics and Graduate School of Pure and Applied Science, University of Tsukuba, Tsukuba 305-8573, Japan

(Received 29 December 2005; accepted 13 March 2006; published online 15 May 2006)

High-efficiency visible light emission in $\mathrm{N}$-and-B-doped $6 \mathrm{H}$-SiC epilayers was observed in photoluminescence measurements at room temperature. The orange-yellow light emission due to the recombination of donor-acceptor pairs (DAPs) has a broad spectrum with a peak wavelength of $576 \mathrm{~nm}$ and a full width at half maximum of $110 \mathrm{~nm}$ at $250 \mathrm{~K}$. The high B concentration of more than $10^{18} \mathrm{~cm}^{-3}$ improves the emission efficiency of the DAP recombination at a high temperature. Compared with the photoluminescence spectrum of $\mathrm{GaN}$ at $10 \mathrm{~K}$, a high quantum efficiency of 95\% was estimated for the highly B-doped sample. From time-resolved photoluminescence measurements, a DAP recombination time of $5.0 \mathrm{~ms}$ was obtained, which is in good agreement with the calculated value by the rate equation with the assumption of a $95 \%$ internal quantum efficiency. This is quite promising as a light-emitting medium by optical pumping, as well as monolithic light sources combined with nitride-based light-emitting diodes grown on the DA-doped $\mathrm{SiC}$ epilayer.

(C) 2006 American Institute of Physics. [DOI: 10.1063/1.2195883]

\section{INTRODUCTION}

White light-emitting diodes (LEDs) are very promising devices for such huge lighting applications such as the backlight source of liquid crystal flat display panels, the headlights of automobiles, and general lighting equipment. Recent advances in nitride-based LEDs have made it possible to realize a white LED with a combination of a blue-LED chip and a yellow phosphor such as yttrium aluminum garnet (YAG):Ce. ${ }^{1}$ These LEDs are already used as a light source in a variety of handheld equipment. The white LED is, in principle, compact, robust, and an efficient light source. Therefore, some people believe that all conventional light sources will be replaced by white LEDs in the future. However, there are still some problems with conventional white LEDs: a low total flux, a low efficiency, and a low color rendering index. These problems are delaying the expansion of white LED applications.

Because the conventional white LED mentioned above emits blue light and yellow light, the color rendering index is

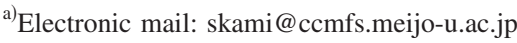

very low due to the lack of red phosphors. Another white LED comprising a UV-LED and three-color phosphors has also been developed to improve the color rendering property. However, this type of device has a low emission efficiency, due to the low efficiency of red phosphors. Thus, the color rendering index and emission efficiency are in a trade-off relationship. In addition, the combination of a singlespectrum LED and phosphors has an intrinsic instability of color against temperature change and divergence angle variation. Moreover, complicated assembly processes are required to set the phosphors uniformly on the LED chip.

Donor-and-acceptor (DA)-doped $\mathrm{SiC}$ seems to be applicable to white LEDs, if it can work as a type of highperformance phosphor, i.e., a light-emission medium by optical pumping. It has many advantages such as a uniform concentration of impurities, excellent thermal conductivity for high-power-operated LEDs, and is a well-established substrate material for nitride growth. Moreover, two types of donor and acceptor pairs (DAPs), N-B and N-Al DAPs, in the $6 \mathrm{H}$-SiC layers can cover almost all the visible spectral range. Therefore, white LEDs with DA-doped $\mathrm{SiC}$ are also 


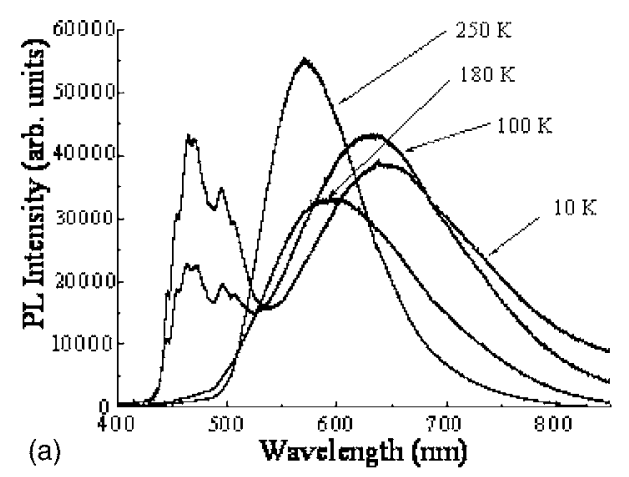

(a)

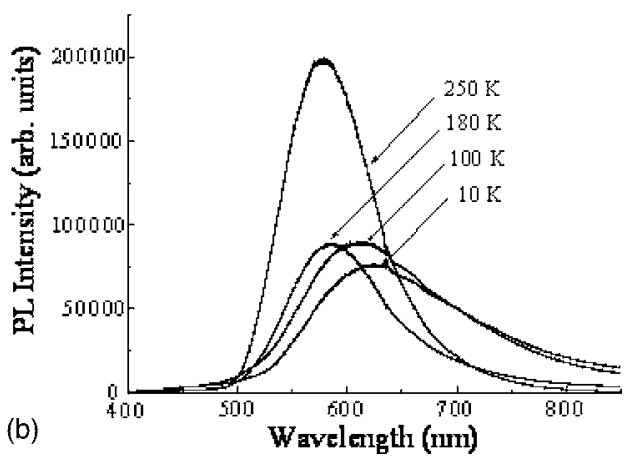

J. Appl. Phys. 99, 093108 (2006)
FIG. 1. Photoluminescence spectra of $\mathrm{N}$-and-B-doped $\mathrm{SiC}$ epilayers. (a) is for sample 1 with low B concentration and (b) is for sample 2 with high B concentration. expected to provide an excellent color rendering index. The key is how high the quantum efficiency of DAP recombination in the $\mathrm{SiC}$ epilayer is.

Many researchers believe that the efficiency of DAP emission in semiconductors is considerably low. This preconception may be proved by the fact that GaP-based red LEDs using DAP emission have quite low external quantum efficiencies on the order of $0.1 \%$. Furthermore, a SiC-based blue LED with a $\mathrm{N}$ (donor)-and-Al (acceptor)-codoped active layer was studied more than 20 years ago, ${ }^{2,3}$ and its efficiency was also very low. Thus, the success of nitride-based blue LEDs at the end of the 1980's (Ref. 4) stopped the development of SiC-based blue LEDs. However, the quantum efficiency of DAP emission itself has not been clarified. Our idea that DA-doped $\mathrm{SiC}$ is used as a passive medium by optical pumping is considerably different from LEDs. The emission rate of DAP in SiC must be much lower than that of an interband transition in direct band gap materials. Therefore, to convert a large number of carriers to photons in the DAP recombination, a large medium volume is indispensable even with a high internal quantum efficiency. This condition cannot be realized in LEDs which require very thin active regions. On the contrary, the light-emission medium by optical pumping basically has no limitation of volume. So, we consider that DAP emission in $\mathrm{SiC}$ is still a promising mechanism toward the realization of white LEDs, if it has a high internal quantum efficiency.

In this paper, we investigated the optical properties of the DAP emission in $\mathrm{N}$-and-B-doped $6 \mathrm{H}$-SiC epilayers. The temperature dependence of the photoluminescence (PL) measurements and time-resolved PL measurements of the DAP recombination were carried out for the estimation of the internal quantum efficiency.

\section{EXPERIMENT}

DA-doped $6 H$-SiC epilayers were grown on commercial $\mathrm{SiC}$ substrates by a closed sublimation technique with a growth temperature of $1900{ }^{\circ} \mathrm{C}$. ${ }^{5}$ The substrates are $n$-type Si-face (0001) $6 \mathrm{H}$-SiC with a $2^{\circ}$ misorientation toward (11-20). Details of the epitaxial growth are shown in the literature. ${ }^{6}$ In two samples, $\mathrm{N}$ concentrations are constant at approximately $4 \times 10^{18} \mathrm{~cm}^{-3}$, and those of $\mathrm{B}$ are $2 \times 10^{17}$ and $2 \times 10^{18} \mathrm{~cm}^{-3}$. The concentrations of impurities were confirmed by secondary ion mass spectroscopy (SIMS) measurements. We define the epilayer with a $\mathrm{B}$ concentration of $2 \times 10^{17} \mathrm{~cm}^{-3}$ as sample 1 and the other with $2 \times 10^{18} \mathrm{~cm}^{-3}$ as sample 2. The reason we used a donor concentration higher than that of the acceptor is to avoid the ionization of donors, which have a small ionization energy of $0.155 \mathrm{eV}$. Due to the high growth rate of the closed sublimation technique, the thicknesses of the epilayers are both more than $100 \mu \mathrm{m}$, which are sufficiently thick compared with the penetration depths of the excitation light sources used in this study.

In the PL measurements, samples were mounted in a cryostat and an unfocused $351 \mathrm{~nm}$ Ar laser was used as an excitation source. The power of the Ar laser was $44 \mathrm{~mW}$, which corresponds to a power density of approximately $2.5 \mathrm{~W} / \mathrm{cm}^{2}$ on the sample surfaces. The temperatures for PL measurement were varied from 10 to $250 \mathrm{~K}$. Figures 1(a) and 1(b) show the PL spectra for two samples at different temperatures, where Fig. 1(a) is for sample 1 and Fig. 1(b) is for sample 2. When the B concentration is low [see Fig. 1(a)], the N-B DAP emission at approximately $630 \mathrm{~nm}$ and the N-Al DAP emission at $460 \mathrm{~nm}$ were simultaneously observed below $100 \mathrm{~K}$. The additional impurity, Al, might be unintentionally introduced from the atmosphere of the growth reactor. Above $100 \mathrm{~K}$, the $\mathrm{N}-\mathrm{Al}$ DAP emission is steeply quenched, and only the N-B DAP emission is observable. The peak wavelength of the N-B DAP emission is approximately $630 \mathrm{~nm}$ at a low temperature, however, it shifts to $576 \mathrm{~nm}$ with increasing temperature. On the other hand, only the N-B DAP emission is observed even at a low temperature in the case of sample 2 [see Fig. 1(b)]. Although the peak shift behavior of the N-B DAP with the temperature variation is similar to the case of sample 1, the intensity significantly increases at a high temperature.

From the PL spectra shown in Fig. 1, the integrated photon counts were calculated. These are shown as functions of temperature in Fig. 2. The two samples exhibit different behaviors, i.e., the integrated photon count tends to decrease with increasing temperature in sample 1, while it significantly increases in sample 2 . At $250 \mathrm{~K}$, sample 2 with a higher B concentration has a PL emission intensity approximately three times higher than sample 1. In addition, the color of the N-B DAP emission in sample 2 seems to be suitable for lighting applications because it is pure orange at a low temperature, and orange yellow at a high temperature. Moreover, the color of the emission of sample 1 at a low temperature looks pure white because of the incorporation of $\mathrm{N}-\mathrm{Al}$ DAP emission.

As a reference, we also measured a $1.5-\mu \mathrm{m}$-thick GaN 


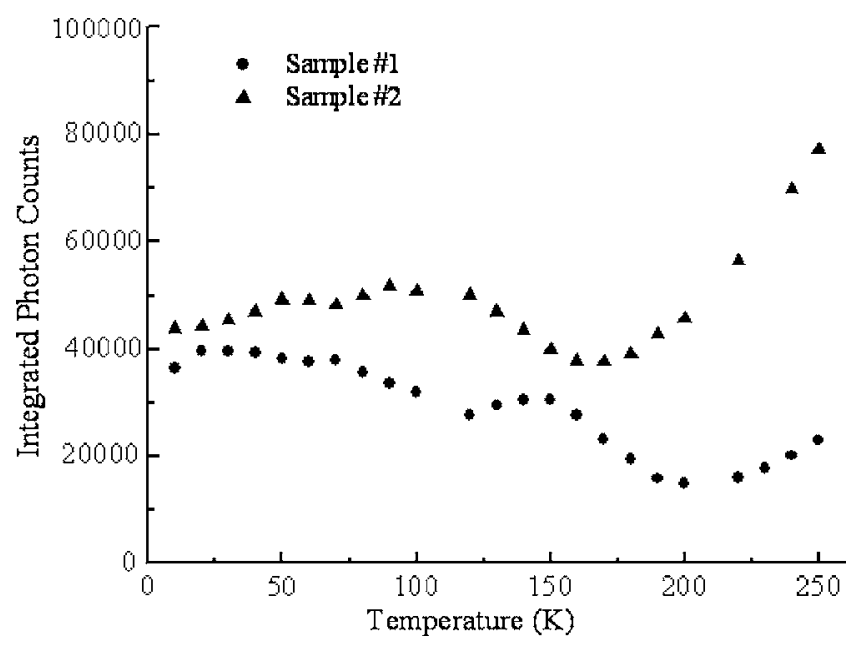

FIG. 2. Integrated photon count of PL spectra as function of temperature.

epilayer grown on a sapphire substrate with the same measurement setup, where a $244 \mathrm{~nm}$ laser was used for the excitation because the absorption edge of the $\mathrm{GaN}$ is too close to $351 \mathrm{~nm}$ at a low temperature. The $\mathrm{GaN}$ and sample 2 were mounted simultaneously into the cryostat. Since the excitation power of the $351 \mathrm{~nm}$ laser for sample 2 of $4.6 \mathrm{~mW}$ and that of the $244 \mathrm{~nm}$ laser for $\mathrm{GaN}$ of $6.4 \mathrm{~mW}$ were used in this measurement, they have almost the same excitation rate (excitation photon number per second). The light extraction efficiencies for $\mathrm{SiC}$ and $\mathrm{GaN}$ seem to be equivalent because of their comparable refractive indices, and the coupling efficiencies of their emissions to the detection system were also the same. Figure 3 shows the integrated photon count for both samples as a function of temperature. The integrated photon count for sample 2 at $250 \mathrm{~K}$ is almost the same as that of $\mathrm{GaN}$ at $10 \mathrm{~K}$. Because $\mathrm{GaN}$ grown on a sapphire substrate contains threading dislocations at a density as high as $2 \times 10^{9} \mathrm{~cm}^{-2}$, it has a very low quantum efficiency at room temperature. However, the nonradiative recombination caused by the dislocations could be eliminated when the temperature is very low. Therefore, the internal quantum efficiency of $\mathrm{GaN}$ at $10 \mathrm{~K}$ may be very high, approximately

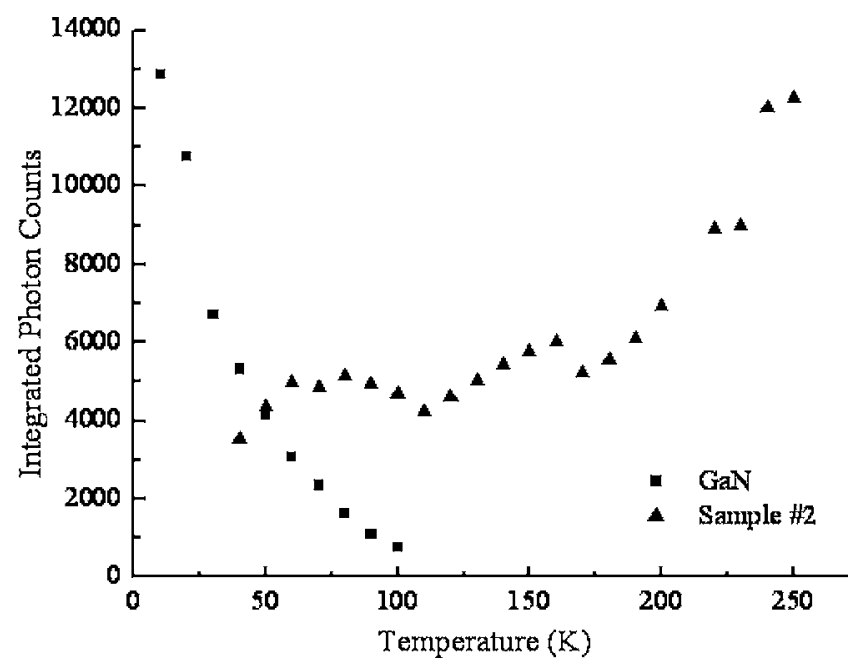

FIG. 3. Integrated photon count of PL spectra for both samples as function of temperature.

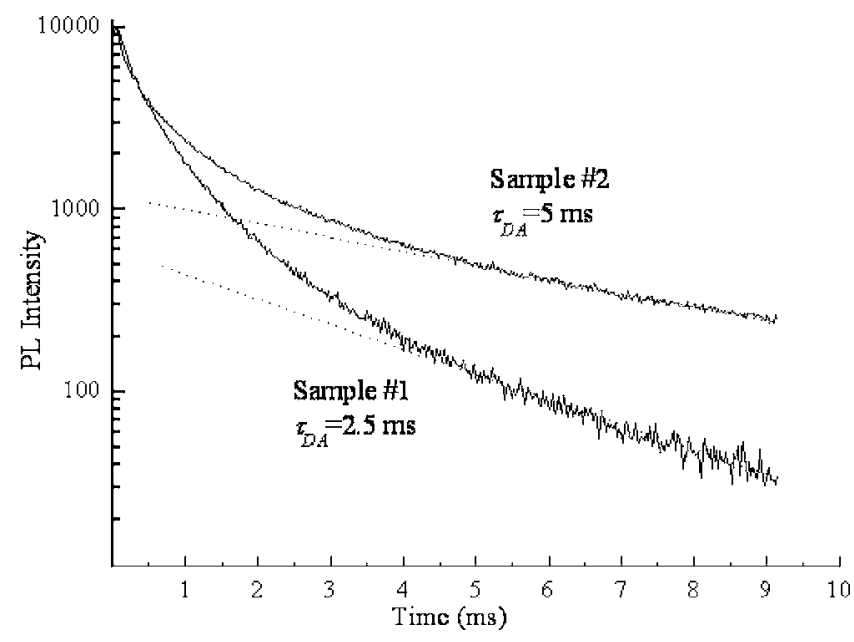

FIG. 4. Semilogarithmic decay curves at room temperature for monochromatic photon energy of $2.15 \mathrm{eV}$. Dotted lines are guides for the eye.

$100 \% .^{7}$ Thus, it should be noted that the internal quantum efficiency of sample 2 at $250 \mathrm{~K}$ is estimated to be $95 \%$.

Time-resolved PL measurements were then carried out. Here, the excitation source was a $355 \mathrm{~nm}$ frequency-tripled YAG:Nd laser excited by a pulsed laser diode. A pulse width of $5 \mathrm{~ns}$ and a power density of $38 \mathrm{~mJ} / \mathrm{cm}^{2}$ pulse were used. The detection was performed using a streak camera with a time resolution of $10 \mathrm{ps}$. Figure 4 shows semilogarithmic decay curves at room temperature for the monochromatic photon energy of $2.15 \mathrm{eV}$, corresponding to the peak of DAP emission. Similar to the case of interband recombination, the monochromatic DAP emission should follow the single exponential decay, because it is caused by the recombination of the electrons at the donor states and holes at the acceptor with a constant distance. We observed several decay time components from the submicrosecond order to the millisecond order. Because of the very high excitation rate in this measurement compared with the static PL measurements, the fast decay component may be due to the nonradiative recombination at the band edge or overlap of another DAP recombination. Approximately $5 \mathrm{~ms}$ after the drop in excitation, the PL intensities follow single exponential decay. These ranges might be under equivalent excitation conditions as those of the static PL measurements shown in Fig. 4. From the decay curves in these ranges, we obtained the DAP recombination times of $2.5 \mathrm{~ms}$ for the low-doping sample and $5.0 \mathrm{~ms}$ for the high-doping sample.

Details about the relationship between the DAP recombination time and the internal quantum efficiency will be discussed in the next section.

\section{RATE EQUATION ANALYSIS}

To evaluate the validity of the high internal quantum efficiencies obtained in Fig. 3, rate equation analysis was carried out in this section. For the consideration of the internal quantum efficiency and rates of carrier relaxations, the band diagram shown in Fig. 5 is useful. Under the thermal equilibrium condition in DA-doped $\mathrm{SiC}\left(N_{D}>N_{A}\right)$, almost all the acceptor states are ionized, as shown in Fig. 5(a). The interband recombination time $\tau_{n}$ is determined by the nonra- 


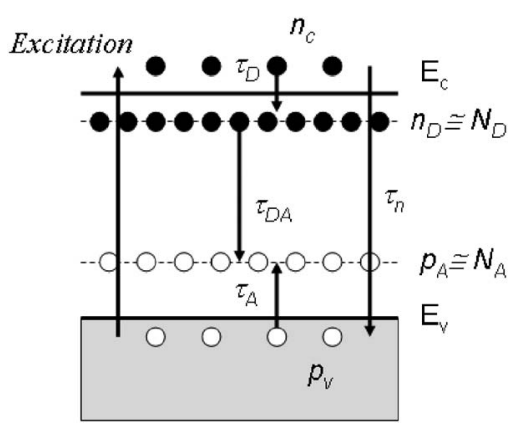

FIG. 5. Band diagrams of DA-doped SiC under excitation conditions, where $N_{D}>N_{A}$.

diative recombination, because $\mathrm{SiC}$ is an indirect band gap material. The DAP recombination time $\tau_{\mathrm{DA}}$ is only determined by the relaxation of holes from the valence band to the acceptor state whose relaxation time is $\tau_{A}$, because of the excess doping of donors in our case. Therefore, the internal quantum efficiency $\eta_{\text {int }}$ and the interband relaxation time $\tau_{\mathrm{BE}}$ are represented by

$$
\begin{gathered}
\eta_{\text {int }}=\frac{1}{1+\tau_{A} / \tau_{n}}, \\
\tau_{\mathrm{BE}}=\frac{\tau_{A} \tau_{n}}{\tau_{A}+\tau_{n}} .
\end{gathered}
$$

From the comparison of integrated PL photon counts, the internal quantum efficiencies of $20 \%$ for sample 1 and $95 \%$ for sample 2 were assumed. Then, we obtained the relationships

$$
\tau_{\mathrm{BE}}=0.2 \tau_{A},
$$

for sample 1 , and

$$
\tau_{\mathrm{BE}}=0.95 \tau_{A},
$$

for sample 2 .

The rate equations for the hole density in the valence band, $p_{v}$, and that in the acceptor state, $p_{A}$, are represented by

$$
\begin{aligned}
& \frac{d p_{v}}{d t}=\frac{I(1-1 / e)}{h \nu d}-\frac{p_{v}}{\tau_{\mathrm{BE}}}, \\
& \frac{d p_{A}}{d t}=\frac{p_{v}}{\tau_{A}}-\frac{p_{A}}{\tau_{\mathrm{DA}}},
\end{aligned}
$$

where $I$ is the excitation power density of excitation $\left(\mathrm{W} / \mathrm{cm}^{2}\right), h$ is the Planck constant, $\nu$ is the frequency of excitation light, and $d$ is the penetration depth of the excitation. The first term in Eq. (5) represents the average excitation rate within the penetration depth. Under the experimental conditions of the static PL measurements in Fig. 3, I was $0.41 \mathrm{~W} / \mathrm{cm}^{2}$ and $d$ was estimated to be $10 \mu \mathrm{m}$. From the equilibrium in Eq. (5),

$$
p_{v}=4.6 \times 10^{20} \tau_{\mathrm{BE}},
$$

was derived. Because the hole density at the acceptor states, $p_{A}$, must be very close to the acceptor concentration $N_{A}$ un- der the excitation, we assumed that $p_{A}$ is equal to $N_{A}$. By substituting Eqs. (3) or (4) and (7) into Eq. (6), we finally obtained the DAP recombination times of $2.2 \mathrm{~ms}$ for sample 1 and $4.6 \mathrm{~ms}$ for sample 2. These values are in good agreement with those obtained in the time-resolved PL measurements shown in Fig. 4.

This agreement means that our estimation of internal quantum efficiencies from the integrated PL photon counts is fairly appropriate. Accordingly, the DAP emission in $6 \mathrm{H}$-SiC has a very low recombination rate, compared with the interband recombination in direct band gap semiconductors. Therefore, it is necessary to use a large volume of donor-and-acceptor-doped medium for a high internal quantum efficiency, which is impossible to realize in current injection devices such as LEDs. The N-and-B-doped $6 \mathrm{H}$-SiC with optimum doping concentrations is a very promising light-emission medium for optical excitation.

\section{CONCLUSION}

By static and time-resolved PL measurements, the optical properties of a $\mathrm{N}$-and-B-doped $6 \mathrm{H}$-SiC epilayer were investigated. When the $\mathrm{B}$ concentration is high, exceeding $10^{18} \mathrm{~cm}^{-3}$, the integrated intensity of the DAP emission increases, in particular, at high temperatures up to $250 \mathrm{~K}$. At $250 \mathrm{~K}$, the observed DAP spectrum has a broad spectrum, where the peak wavelength is $576 \mathrm{~nm}$ and the full width at half maximum is $110 \mathrm{~nm}$. Compared with the integrated PL intensity of $\mathrm{GaN}$ at $10 \mathrm{~K}$, that of $\mathrm{N}$-and-B-doped $\mathrm{SiC}$ at $250 \mathrm{~K}$ was estimated to be $95 \%$. From time-resolved PL measurements, the DAP recombination time was obtained to be $5.0 \mathrm{~ms}$. This value is in good agreement with that obtained from the rate equation analysis with the assumption of a $95 \%$ internal quantum efficiency. The DA-doped $6 H$-SiC is promising for a light-emitting medium using optical pumping, as well as monolithic light sources combined with nitride-based light-emitting diodes.

\section{ACKNOWLEDGMENTS}

This work is supported by the "Creation and Support Program of Startups from Universities," from the Japan Science and Technology (JST) Agency. One of the authors (S.K.) would like to thank Dr. P. P. Paskov at Linköping University, Sweden, for fruitful discussions.

\footnotetext{
${ }^{1}$ Y. Narukawa, I. Niki, K. Izuno, M. Yamada, Y. Murazaki, and T. Mukai, Jpn. J. Appl. Phys., Part 2 41, L371 (2002).

${ }^{2}$ H. Matsunami, M. Ikeda, A. Suzuki, and T. Tanaka, IEEE Trans. Electron Devices ED-24, 958 (1977).

${ }^{3}$ M. Ikeda, T. Hayakawa, S. Yamagiwa, H. Matsunami, and T. Tanaka, J. Appl. Phys. 50, 8215 (1979).

${ }^{4}$ H. Amano, M. Kito, K. Hiramatsu, and I. Akasaki, Jpn. J. Appl. Phys., Part 2 28, L2112 (1989).

${ }^{5}$ S. Nishino, K. Matsumoto, T. Yoshida, Y. Chen, and S. K. Lilov, Mater. Sci. Eng., B B61-62, 121 (1999).

${ }^{6}$ Y. Kawai et al., Proceedings of the ICSCRM, 2005 (unpublished).

${ }^{7}$ J. S. Im, A. Moritz, F. Steuber, V. Harle, F. Scholz, and A. Hangleiter, Appl. Phys. Lett. 70, 631 (1997).
} 